\title{
Influencia del contenido de OHAP y de gentamicina en la liberación del antibiótico a partir de composites de OHAP/PEMMA
}

\author{
S. PADILLA ${ }^{1,2}$, R.P. DEL REAL ${ }^{2}$, M. VALLET-REGí \\ ${ }^{1}$ Dpto. Cerámicas y Composites, Centro de Biomateriales, \\ Universidad de La Habana, Ciudad de La Habana 10400, Cuba \\ ${ }^{2}$ Dpto. Química Inorgánica y Bioinorgánica, Facultad de Farmacia, \\ Universidad Complutense, Madrid 28040, España
}

\begin{abstract}
Resumen
El objetivo de este trabajo fue estudiar la influencia del contenido de hidroxiapatita (OHAp) y de sulfato de gentamicina ( $S G)$ en la velocidad de liberación de dicho antibiótico a partir de materiales compuestos por OHAp, polimetacrilato de metilo (PMMA) y polimetacrilato de etilo (PEMA).

Para ello se prepararon muestras con diferente proporción de OHAp (30 y 40\%) y de SG (5 y 10\%). El estudio de liberación se llevó a cabo sumergiendo 3 muestras de cada grupo en SBF a $37^{\circ} \mathrm{C}$, el medio fue renovado a diferentes intervalos de tiempo durante 70 días. La concentración de gentamicina fue determinada por el método del o-ftaldialdehído.

Los resultados del estudio de liberación mostraron, que la liberación ocurre de manera más rápida durante las primeras 10 horas de inmersión en SBF y que posteriormente se hace más lenta. Se observó que las muestras con un mayor tanto por ciento (40\%) de OHAp liberan mayor cantidad de antibiótico. Las muestras con un 40\% de OHAp liberaron el 100\% de SG a los 70 días, mientras que en ese mismo período las muestras con $30 \%$ de OHAp liberaron un $85 \%$. Por otra parte, se observó que la cantidad de gentamicina añadida inicialmente no afecta el tanto por ciento de SG que se libera.
\end{abstract}

\section{Summary}

In this work the influence of the content of hydroxyapatita (OHAp) and gentamicina sulfate (SG) in the release kinetics of this antibiotic in samples composed by OHAp, poly(methyl methacrylate) (PMMA) and poly(ethyl methacrylate) (PEMA) has been studied.

Samples with different proportion of OHAp (30 and 40\%) and of gentamicina (5 and 10\%) were prepared. The release study was carried out soaking three samples of each group into simulated body fluid $S B F$ at $37^{\circ} \mathrm{C}$, the medium was periodically repleced during 70 days. The $S G$ concentration was determined by the o-phtaldialdehyde method.

The release profile shows a faster SG release during the first 10 hours, after that it becomes slower. The samples with a larger percent of OHAp $(40 \%)$ release higher quantity of antibiotic. The samples with $40 \%$ of OHAp liberated 100\% of SG in 70 days, while the samples with $30 \%$ of OHAp liberated $85 \%$ in the same period. On the other hand, it was observed that the quantity of SG added initially doesn't affect the percent of SG that is released.

Correspondencia:

María Vallet-Regí

Dpto. Química Inorgánica y Bioinorgánica,

Facultad de Farmacia,

Universidad Complutense, Madrid 28040, España

E-mail: vallet@eucmax.sim.ucm.es 


\section{Introducción}

La aplicación de biomateriales compuestos cerámica-polímeros, ha alcanzado gran importancia en los últimos años, ya que de esta manera se logra combinar las ventajas de cada uno de los materiales por separado. Además, éstos pueden emplearse como matriz a la cual pueden incorporarse fármacos, tales como, antibióticos, analgésicos, antinflamatorios, antitumorales, anticancerígenos, etc..

Dentro de las cerámicas, las de fosfatos de calcio, fundamentalmente las de hidroxiapatita y $\beta$ fosfato tricálcico, han sido las más utilizadas, en la restauración del sistema óseo, debido a la alta biocompatibilidad y bioactividad que estas presen$\tan$.

En este trabajo se prepararon materiales compuestos por hidroxiapatita (OHAp), polimetacrilato de metilo (PMMA) y polimetacrilato de etilo (PEMA), utilizando peróxido de benzoílo $\left(\mathrm{PBO}_{2}\right)$ y alcohol-4[N,N dimetilaminobenzílico] (DMOH) como par iniciador/activador de la polimerización, de forma que el fraguado ocurre a temperatura ambiente. Como fármaco se añadió gentamicina, un antibiótico del grupo de los aminoglicósidos. Se estudió la influencia del contenido de OHAp y de gentamicina, en la velocidad de liberación de dicho fármaco.

\section{Materiales y métodos}

\section{Preparación de los materiales compuestos}

Los materiales compuestos fueron preparados a temperatura ambiente por polimerización radicálica de una mezcla de OHAp, PEMA, SG y MMA, iniciada por el sistema $\mathrm{PBO}_{2} / \mathrm{DMOH}$. Pri- meramente, se mezclaron los componentes sólidos del sistema (OHAp, PEMA, SG, $\mathrm{PBO}_{2}$ ) y posteriormente se adicionó una solución de $\mathrm{DMOH}$ en MMA (componente líquido). Se prepararon muestras con diferente proporción de OHAp (30 y 40\%) y de SG (5 y 10\%). La composición de los materiales compuestos estudiados se muestra en la siguiente tabla.

\section{Estudio de liberación in vitro}

Para el estudio de liberación de gentamicina in vitro, se sumergieron tres muestras de cada grupo en $5 \mathrm{~mL}$ de fluido biológico simulado (SBF, $\mathrm{pH}=7.25)$ y se mantuvieron, durante el estudio, a $37^{\circ} \mathrm{C}$ en estufa. El SBF empleado fue filtrado antes de añadirlo en los frascos de estudio (tamaño de poro $0.22 \mu \mathrm{m}$ ). Las muestras fueron suspendidas con alambres de platino de forma que quedaran verticales y no tocaran el fondo del frasco, con el objetivo de que toda la superficie del cuerpo estuviera expuesta al medio. Durante el estudio el SBF fue renovado a diferentes intervalos de tiempo, la renovación del líquido se realizó en campana de flujo laminar. El estudio de liberación de gentamicina se siguió durante 70 días.

La determinación de la concentración de gentamicina se realizó mediante espectroscopía UV-VIS, utilizando o-ftaldialdehído como reactivo derivatizante. Para poder realizar la determinación de gentamicina por este método fue necesario realizar una modificación al SBF reportado por Kokubo et al. ${ }^{1}$, pues el tris-hidroximetilaminometano que se añade como tampón, interfiere en la determinación de la concentración de sulfato de gentamicina.

El complejo formado entre la gentamicina y el o-ftaldialdehído se leyó a una longitud de onda $\lambda=331.5 \mathrm{~nm}$ en un espectrofotómetro Perkin Elmer

Tabla 1. Composición de los materiales compuestos.

\begin{tabular}{c|cc|cc}
\hline \multirow{2}{*}{$\begin{array}{c}\text { Componentes } \\
\text { (\% en peso) }\end{array}$} & \multicolumn{2}{|c|}{ Serie A } & \multicolumn{2}{c}{ Serie B } \\
\cline { 2 - 5 } OHAp & G. A1 & G. A2 & G. B1 & G. B2 \\
PEMA & 30 & 30 & 40 & 40 \\
MMA & 40 & 30 & 25 & 25 \\
SG* & 5 & 10 & 35 & 35 \\
\hline
\end{tabular}

*Los porcientos de SG añadidos están calculados con respecto a la suma de las masas de OHAp, PEMA y MMA 


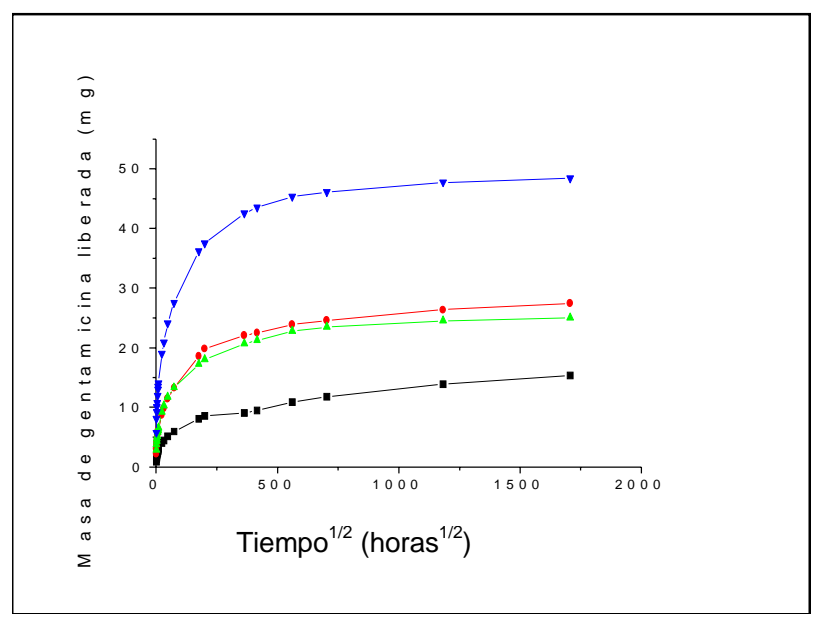

Figura 1. Masa de gentamicina liberada con respecto al tiempo

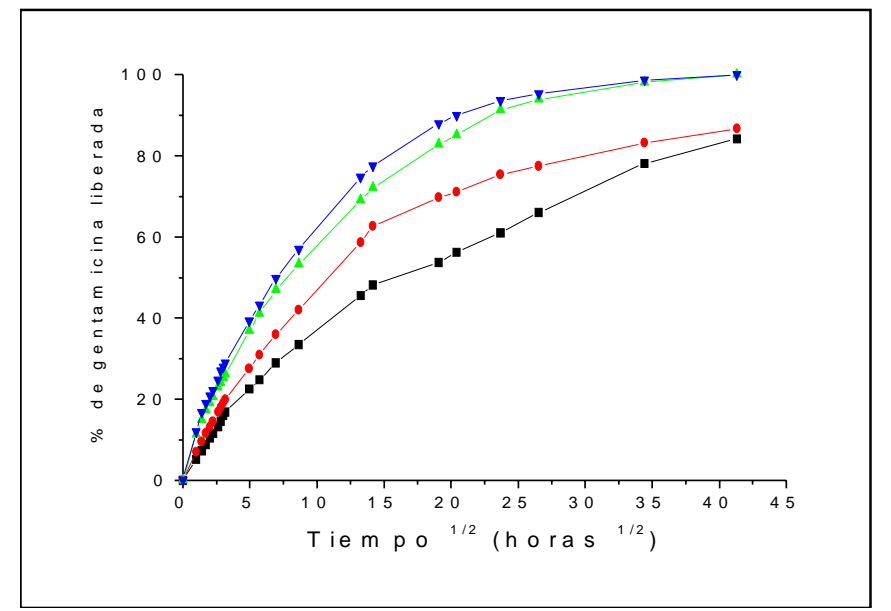

Figura 2. Tanto por ciento de gentamicina liberada con respecto a la raíz cuadrada del tiempo.

554. Previamente se realizó una curva de calibración, siendo los parámetros de la recta de calibrado obtenidos similares a los reportados por los autores de este método².

\section{Resultados y discusión}

\section{Estudio de liberación in vitro}

El perfil de liberación de gentamicina en SBF se muestra en la Figura 1 donde se representa la liberación de gentamicina en función de los días de inmersión. En ella se observa que durante las primeras 10 horas del estudio la liberación es más rápida, disminuyendo la velocidad hasta los 16 días aproximadamente y luego se hace mucho menor hasta el final del estudio (70 días).

En la Figura 2 aparece representado el tanto por ciento de SG liberado con respecto a la raíz cuadrada del tiempo, en ellos se observa que las mues- tras preparadas con un $30 \%$ de OHAp liberan un $85 \%$ de SG al final del estudio, mientras que aquellas en que se añadió un $40 \%$ de SG liberaron el $100 \%$ del SG añadido. Por otra parte, la cantidad de SG añadida no afecta el tanto por ciento del antibiótico que se libera.

El sistema en estudio está constituido por una matriz orgánica en la que se ha incluido una cierta cantidad de gentamicina y en cuyo seno se ha dispersado OHAp como componente inorgánico. Al representar la fracción de gentamicina liberada con respecto a la raíz cuadrada del tiempo (Figura 2) se observa que los resultados se ajustan a un polinomio de tercer grado, lo que corresponde con la ecuación propuesta por Cobby et al. ${ }^{3}$ para este tipo de sistemas (heterogéneos, con matriz bioestable y con canales y poros), con piezas de forma cilíndrica donde toda la superficie se encuentra expuesta al medio de liberación. 
La ecuación que modela este comportamiento es la siguiente:

$$
\begin{gathered}
f_{r}=(q+2) K_{r} \sqrt{t}-(2 q+1)\left(K_{r} \sqrt{t}\right)^{2}+q\left(K_{r} \sqrt{t}\right)^{3} \\
q=\frac{r_{o}}{h_{o}}, \\
K_{r}=\frac{K_{b}}{r_{o}}=\frac{2}{r_{o}} \sqrt{C_{s} \frac{D \in}{\tau\left(2 C_{e}-\in C_{s}\right)}}
\end{gathered}
$$

donde: $\mathrm{f}_{\mathrm{t}}$ es la fracción del fármaco liberado en el tiempo $t, \mathrm{~K}_{\mathrm{r}}$ la constante de liberación, $\mathrm{r}_{0}$ el radio inicial del material compuesto, $\mathrm{h}_{0}$ la mitad de la altura, $\mathrm{K}_{\mathrm{b}}$ la constante de disminución de la frontera, $\mathrm{D}$ el coeficiente de difusión, $\mathrm{C}_{\mathrm{s}}$ la solubilidad del fármaco en el medio de estudio, $\mathrm{C}_{\mathrm{d}}$ la masa inicial de fármaco en el compuesto, $\in$ la porosidad y $\tau$ la tortuosidad.

En este caso la liberación de gentamicina ocurre por un mecanismo de difusión, donde en primer lugar se libera el fármaco que está en la superficie y en los lugares más accesibles y posteriormente el antibiótico difunde al medio a través de los canales y poros de la matriz así como por los que va dejando el fármaco al disolverse.

\section{Conclusiones}

La adición de un mayor contenido de hidroxiapatita a los materiales compuestos por
OHAP/PEMA/PMMA trae consigo una mayor liberación de sulfato de gentamicina, mientras que la cantidad del antibiótico que se añade no afecta el tanto por ciento que se libera.

Según esto, la cantidad de gentamicina que se libera de estos materiales puede ser regulada variando la cantidad de hidroxiapatita y de fármaco que se añade.

\section{Agradecimientos}

Los autores agradecen al Proyecto de Investigación Conjunta ICI-MEC/PR264/97 y al CICYT por el financiamiento a través del proyecto MAT960919. R. P. del Real agradece además, a la Consejería de Educación y Cultura (C.A.M.) por su beca postdoctoral.

\section{Referencias}

1. Kokubo T, Kushitani H, Sakka S, Kitsugi T, Yamamuro T. Solutions able to reproduce in vivo surface-structure changes in bioactive glass-ceramic A-W³. J Biomed Mater Res 1990;24:721-734.

2. Sampath SS, Robinson, DH. Comparison of new and existing spectrophotometric method for the analysis of tobramycin and other aminoglicosids. J Pharm Sci 1990;79:428-431.

3. Cobby J, Mayersohn M, Walker GC. Influence of shape factors on kinetics of drug release from matrix tables I: Theorical. J Pharm Sci 1974;63:725-732 